\title{
From Shen Congwen's Work "Xiao Xiao" to Unscramble the Core Value of Chinese Language and Literature
}

\author{
Xinyan Li \\ Zhengzhou University of Science and Technology, Zhengzhou, Henan, 450064, China
}

Keywords: Xiao Xiao, Western Hunan, Language expression, Slang vocabulary, Rhythm

\begin{abstract}
Xiao Xiao” is one of Shen Congwen's representative work, it is like a piece of beautiful bright pastoral, in simple and beautiful humanity light shines, but brought a kind of difficult to queer doubt and sorrow for readers. Although the length of "Xiao Xiao" is short, but the language characteristic is distinctive, it is this special and independent rural language style brings readers into the western Hunan world by Shen Congwen. It is pure, fresh and simple, language thoughts reveals a strong region nostalgia customs and western Hunan people simple and tough nature of life, makes people drunk simple and beautiful western Hunan country language at the same time also will sigh humanity desolate and the vicissitudes. In this paper, by the description of language world of "Xiao Xiao" to taste of Shen Congwen's language using characteristics, and excavate Chinese language and literature value contained in his work.
\end{abstract}

\section{Introduction}

"Xiao Xiao" depicts a western Hunan girl with tragic fate, shows the fate of humanity desolate in the old society of Chinese feudal system. Language depict in the work is close to human feelings even to human nature, fully display out the three stages affect Xiao Xiao's fate, also associated with strong social conflicts and human eulogium. "Newly Organized College Chinese" evaluated and thought "the language in "Xiao Xiao" rooted in the soil of western Hunan life; in the pristine it is a mixture of narrative about the living and life of countryside language.” Shen Congwen not only showed the western Hunan's rural and beautiful picture by language, also with language exposed the survival real world images of the western Hunan people at the bottom, the dual character of language use also showed Shen Congwen's excels as “writing magician”.

\section{The voice of western Hunan migrated in the tone}

In the language of expression of "Xiao Xiao", most differences reflected by phonetic form structure, such as dialect. Use of dialect also was the most important creation means of Shen Congwen, though in past writers, the dialect into the expression means of the text was not enough and the difference was not clear, but in the "Xiao Xiao", western Hunan dialect was very harmoniouswith language text, it was profit from the author's profound understanding on western Hunan's rural language and the world of western Hunan itself.

For example, in "Xiao Xiao" there were many dialect markers, such as tail words: niu-er, zhipian-er and so on, as well as sub tail word: ri-zi, shoubang-zi, xinqiao-zi, banggonghan-zi and so on. Moreover, the tail words and the sub tail words were used paralleling in the article, which was also the true representation of the characteristics of the western Hunan dialect. In addition, there were many dialect examples revealed in spite of the authors, such as:

"No matter somehow, to be a female student is not terrible, therefore, in this way the country has the consideration."

The word "consideration" in the statements is typical performance of the western Hunan dialect, although there are two entries of "consideration" and "experience" in the "Modern Chinese words dictionary", but the expression means are markedly different. "Consideration" has the meaning of considering for others in western Hunan dialect. In Chinese, "experience" is through the practice to perceive the surrounding things, to achieve the effect of personal experience. The two words are closer on the meaning in the language, and also the same pronunciation in Chinese. 
Then from the modal particle at the end of the statement to analyze, the modal particle often attached at the end of the words in "Xiao Xiao", that it expressed some kind of mood of the actual situation, so modal particle is an important symbol of the language and speech prosody in the works. This has different approaches but equally satisfactory results to modal particle "xi" in ancient Chinese Chuci "write in Chu language, speak Chu dialect", both of them can express melodious lingering special flavor in language. And this kind of tone with a large number of wetern Hunan dialect characteristic modal particles in "Xiao Xiao", originally expressed unique narrative rhythm and rhythm of "Xiao Xiao". Here is another example:

"We go to the city to be more free, help others to live, that is not good?"

"You want to escape to the city to be free, not to be!"

In the statement, "de" is a typical western Hunan Mandarin tone word. And "me" is typical of the western Hunan dialect tone words, both of them were interdependent coexistence state in western Hunan dialect, and the two modal particles in the language flow formed promotion and demotion tone could also reveal the unique and charming accent rhythm of western Hunan dialect, increased emotional expression for the work.

Shen Congwen as realistic writers lived in western Hunan for long, he also had the consciousness of the mobilization of the sensory dialect of the language works which were most likely to be aware of, the mood of western Hunan dialect image was unnatural built up in the work, and blended by dialect world, showed a vivid and rich western Hunan dialect world for people. Take the following article For example:

"The thing of female student is always a stranger in the countryside. Those June days are "water holidays", and then as usual there will be several female students, from a ridiculous lively place, to another distant place, via the local for across.”

In this paragraph, Shen Congwen used the term "water holidays", which not only retained the unique features of western Hunan area, but also manifested the kind of cognitive attitude towards things ${ }^{[1]}$.

\section{The use of slang words and the cognitive concept of roles in "Xiao Xiao"}

It should be said that the "voice" can only reflect part of the text language use of the work, to determine whether "Xiao Xiao" was a real mature work, should also judge from the unique language use of the work. And in "Xiao Xiao", there was exactly a unique words expression system- the rural village language. The rural village language in "Xiao Xiao" had strong regionalism, it conveyed western Hunan local village people's cognition, understanding and imagination to the world, also these constituted the complete map of "Xiao Xiao" world, there were sketches of western Hunan regional customs, also western Hunan people's sense of thoughts about the closed, idyllic beauty like society. So we say that western Hunan people were frugal, Shen Congwen with his style of writing and reproduced the closed world, in which the most important expression tool was literary language, its characteristic was true and simple.

Analysis of the western Hunan characteristic vocabulary based on text classification. In the text of "Xiao Xiao", all kinds of words expressed their functions, such as table 1

Table 1. The use of nouns, adjectives and verbs in "Xiao Xiao"

\begin{tabular}{cccc}
\hline parts of speech / items & quantity & proportion & example \\
\hline noun & 1015 & $51.3 \%$ & country folk, thrum $\cdots \cdots \cdot$ \\
\hline verb & 705 & $36.0 \%$ & Crawl, shout, coax $\cdots \cdots \cdot \cdots$ \\
\hline adjective & 261 & $12.7 \%$ & Unfamiliar, palatial, joyful $\cdots \cdots$ \\
\hline totalize & 1981 & $100 \%$ & \\
\hline
\end{tabular}

From table1 we can see, the most use of noun in the text of "Xiao Xiao", more than half of the total vocabulary amount of the text, while the verb and adjective was less. From the perspective of the theoretical analysis of the Chinese language, if take the language as the carrier of thinking, then the noun reflect the cognitive subject, in this includes the cognition of people or things. What verb expresses is that the entire cognitive subject's behavior, body movements and mental activities, and 
they may change may also disappear. And the adjectives in the works were few and the essence, only appeared words could be quite accurately express the character roles' language cognitive state, experience the feeling and the nature of things. Although the two categories of nouns and verbs can construct into sentence pattern and relatively simple language communication, the adjective can further deepen and enrich human cognition and the plot world. In the text of "Xiao Xiao", the strong contrast between the three kinds of vocabulary were also reflect people's simple, rough lines perspective in the western Hunan world from a layer, if combined with the article to taste will feel more obvious.

At the beginning, "at the end of the year western Hunan local people play suona to greet wife is an everyday occurrence." In this sentence, in addition to adhesion function word "de" and "le", other words full composed of nouns and verbs, litterateur called the expression of this style of Shen Congwen as "white description in western Hunan language", although it was simple but draws the outline of the true love in the world. And in the work, there was such a love song depicted love:

"Clouds in the sky like flower, pod, and plant pod in the bad corn tree, pod wrapped around the corn, and sister wrapped around the brother. Clouds in the sky are heavy, graves buried in the earth are duplicate, the sister washed the bowls, sisters on the bed and the man on her." Although the folk was long but it did ont use an adjective in "Xiao Xiao", instead were a large number of nouns, and they were familiar things and phenomena by western Hunan country people, from the simple life vocabularies, the work also simply but profoundly interpreted plain and simple understand to love in western Hunan country people's heart.

The details of the noun. As mentioned above, the noun is the main melody of the work "Xiao Xiao", the whole text had 351 entries, which western Hunan dialect nouns accounted for the total 3/5, they were the most basic form of slang expression in the western Hunan rural village language. In the use of these words, the use of characters and plant nouns occupied a dominant position. This phenomenon revealed the essence of Shen Congwen's creation of "Xiao Xiao", that was, through the western Hunan rural people to reflect the "the world of rural people", and for the western Hunan, animals, plants and human also constituted the whole world of western Hunan. Such as "water holiday", "fine linen", "ubac", "virgin" and so on all manifested the country people's view of the world. And Shen Congwen also made comparison of life cognitive concept between urban and rural areas people by contrasting some words, such as:

"Many civilized people in cities, spend the whole summer on soft silk clothes, fine drinks and a variety of good things. Xiao Xiao's family, through a summer's work, received more than ten pounds of fine linen, and twenty or thirty loads melon.”

This sentence profoundly reflected the gap between urban and rural areas in the life, and the use of words in the corresponding to the people's language habits. In Shen Congwen's rural village language, the expression of city and country people in many words were not the same, such as movie tickets in the western Hunan's rural population was called "little squares of paper", called movie as "shadow play", called the car as "big box" and so on. So in this literary work, the reader can see another set of language expression system, which was the expression way of the country people in western Hunan. Their expression was self - sufficient and impeccable, and Shen Congwen only from the point of view of literature to the truly restored the daily life of the people in western Hunan, reflected back their true state of life. With the western Hunan dialect, the author also wanted to express a simple understanding of the world, such as the "baby", "virgin" and so on, the use of these words this paper intercepted a typical scene of language expression in "Xiao Xiao".

"At the end of the year western Hunan local people play suona to greet wife is an everyday occurrence. Behind the suona there is a bridal sedan chair, woman is brass lock inside the sedan, although wear decent clothes cannot wear at ordinary times, still heh-heh cry. In the minds of these little women, a bride, away from her mother, then to be a mother, there will be many new things for them, like a dream. "

This description was the description of western Hunan country marriage in the work, although the overall vocabulary was lackluster performance, but were commonly used words by western Hunan underlying rural people, which also corresponding to language scholar's theory "each world view has 
a language expression way”. This paragraph also completely and purely covered the concept and imagination of western Hunan rural human, which was a complete language system. Shen Congwen was more about western Hunan country life, he deeply knew language these words of "play the suona, lift sedan, heh-heh cry" can make works for the occasion, reflected the true life scenes of western Hunan and Xiao Xiao, so it applied these rural village language distinguished in the modern literary world, with exquisite slang and local culture directly and deeply introduced readers to his constructed literature world ${ }^{[2]}$.

\section{The natural use of language}

The work “Xiao Xiao" used simple and natural language, not disobeyed the human nature, also this was Shen Congwen's own highest pursuit of life. He gave this life character to Xiao Xiao, so that she was more natural and vivid in the linguistic hedge of the work.

Western Hunan people loved to sing, it is said that the people in western Hunan was more difficult talking than singing, this was not false. In the work, Huagou used song to pass his love, this was the most true western Hunan style expression, also was plot clue of the article. Huagou moved Xiao Xiao by this way, let her married him. Shen Congwen expressed in this language expression:

"And then one day, Xiao Xiao sang and moved Huagou's heart, and to be a woman."

And in the whole work, metaphor was the use of language and the most direct expression of visualization by Shen Congwen, the metaphors showed people"s value and emotional orientation, and became a humanized rhetoric format, such as this paragraph:

"Although mother-in-law born like a pair of scissors, cut all opportunities of sudden bursts of Xiao Xiao, but the sun and air of the country help people grow up, cannot be obstructed by torture. When Xiao Xiao was fifteen years old, she was as tall as an adult, but she still had a very vague heart."

In this expression, the metaphors were numerous, but also simple. The bottom of the western Hunan granted their habitual things to make metonymy of live and life, and described their known world, so that these metaphors were grounded, vivid, rich local color and flavor of life. In particular, metaphor mother-in-law as a pair of scissors, visually and vividly outlined her mother-in-law's acridness also traditional character, which also was Shen Congwen's deep understanding of folk language and profound literary skills from living experience accumulation in rural areas for many years $^{[3]}$.

\section{Summary}

Without language, people's hearts will not exist any object. This sentence can be used to evaluate Shen Congwen's literary world, he was such a writer used language and psychology to reflect the literature and show reality, which was vividly reflected in "Xiao Xiao". From the perspective of language ontology, the "western Hunan world” created by "Xiao Xiao" was a success, and the key of success was the use the rural village language. It not only restored the true life of the bottom in western Hunan country at that time, but also reflected the core value in literature of Chinese language.

\section{References}

[1] Wang Kai. The analysis of language rhetoric and rural language in Shen Congwen's work “Xiao Xiao” . Short stories, 2015,4 (10), 35-37.

[2] Wu Shijuan. The analysis of language features and formation causes in Shen Congwen's the western Hunan works. Central China Normal University, 2007.4-25.

[3] Sun Ye Lin. "Western Hunan world” in rural village langyge - new theory on Shen Congwen's novel “Xiao Xiao” . Research of Chinese literature, 2009, (2): 119-122. 